\author{
To reflect the current surge of interest in photonics, we are launching a new Nature- \\ branded journal dedicated to reporting the best research in the field. Nature Photonics is \\ open for business.
}

Welcome to the first issue of Nature Photonics, a new monthly journal from the Nature Publishing Group. Our aim is to publish top-quality research that we believe will excite, inspire and stimulate the optics community.

We have deliberately decided to cover a wide range of topics within the field of photonics, ranging from fundamental new insights into quantum optics through to more device-oriented work. We are also very broad in a geographical sense with editors placed around the globe (in Tokyo, San Francisco and London) - allowing us to more easily visit local labs, attend conferences and interact with scientists.

As editors, we look for the most important findings that involve photons with a wavelength spanning anywhere from the extreme UV to the terahertz region. Our mission is in fact very simple - to bring you ground-breaking results that offer a valuable insight into how light behaves, describe an innovative technology, or report a breakthrough in device performance or functionality.

The content of our first issue illustrates this point well with a combination of papers that cover important advances in optical buffer memory, polarizationtolerant microphotonics, apparatus for mapping the vector field of light, microdisk lasers, and the manipulation of light inside photonic crystals. This is complemented by a review article on the exciting topic of making metamaterials with a negative refractive index, a commentary on the emerging topic of DNA photonics, and much more besides.

Given the strong application-driven nature of photonics it seemed inappropriate to launch a journal that ignores this side of the field. As a result, there is a lot more to Nature Photonics than just important research papers. We will also be having articles looking at emerging applications, technology commercialization and the business side of photonics. Although the topics will vary from month to month, the idea is to bring you up to date with exciting developments in an accessible, easy-toread and independent manner.

To help support this goal, we are publishing a regular series of Technology Focus supplements. Each will pull together a unique selection of articles looking at the status of a particular photonic technology that we believe will be increasingly important in the future. The first of these can be found on p23 and is dedicated to the topic of LEDs. It describes the development of the technology, their role in the automotive industry, progress in making white-emitting organic devices and a round up of recent business news and product launches.

Now, that's enough from us. Enjoy the first issue and don't forget to send us your very best research.

\section{LEFT TO RIGHT}

CHIEF EDITOR: OLIVER GRAYDON ASSOCIATE EDITOR: AMBER JENKINS ASSOCIATE EDITOR: RACHEL PEI CHIN WON ASSOCIATE EDITOR: DAVID GEVAUX
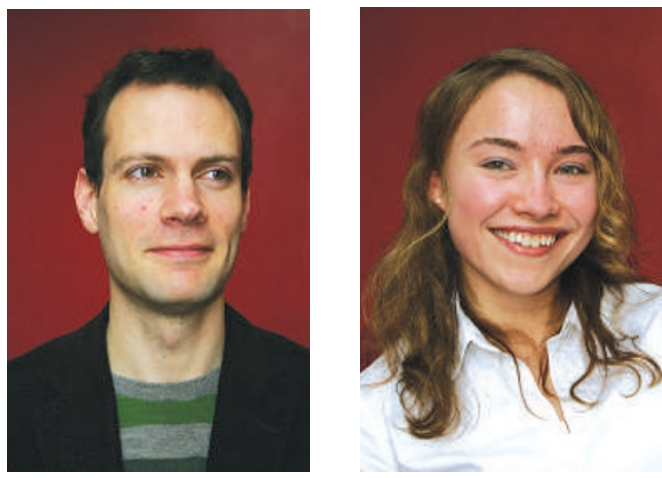
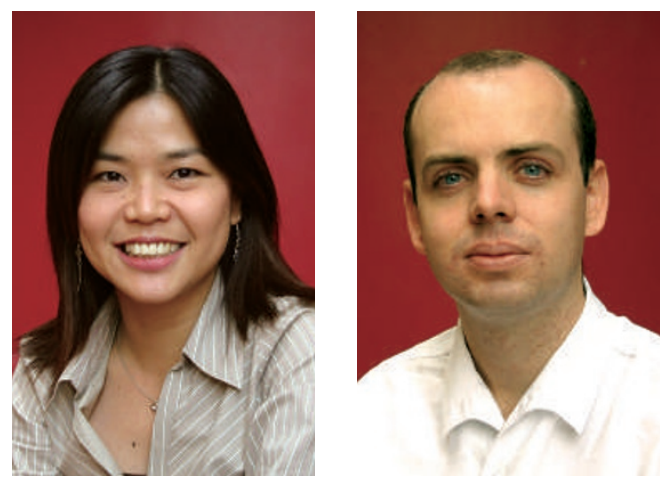